\title{
BOUNDARY VALUE PROBLEMS FOR THE 2ND-ORDER SEIBERG-WITTEN EQUATIONS
}

\author{
CELSO MELCHIADES DORIA
}

Received 8 June 2004

It is shown that the nonhomogeneous Dirichlet and Neuman problems for the 2nd-order Seiberg-Witten equation on a compact 4-manifold $X$ admit a regular solution once the nonhomogeneous Palais-Smale condition $\mathscr{H}$ is satisfied. The approach consists in applying the elliptic techniques to the variational setting of the Seiberg-Witten equation. The gauge invariance of the functional allows to restrict the problem to the Coulomb subspace $\mathscr{C}_{\alpha}^{\mathfrak{C}}$ of configuration space. The coercivity of the $\mathscr{S}^{\mathfrak{W}} \mathcal{W}_{\alpha}$-functional, when restricted into the Coulomb subspace, imply the existence of a weak solution. The regularity then follows from the boundedness of $L^{\infty}$-norms of spinor solutions and the gauge fixing lemma.

\section{Introduction}

Let $X$ be a compact smooth 4-manifold with nonempty boundary. In our context, the Seiberg-Witten equations are the 2nd-order Euler-Lagrange equation of the functional defined in Definition 2.3. When the boundary is empty, their variational aspects were first studied in [3] and the topological ones in [1]. Thus, the main aim here is to obtain the existence of a solution to the nonhomogeneous equations whenever $\partial X \neq \varnothing$. The nonemptiness of the boundary inflicts boundary conditions on the problem. Classically, this sort of problem is classified according to its boundary conditions in Dirichlet problem (D) or Neumann problem $(\mathcal{N})$.

Originally, the Seiberg-Witten equations were described in [8] as a pair of 1st-order PDE. The solutions of these equations were known as $\mathscr{S}^{q} W_{\alpha}$-monopoles, and their main achievement were to shed light on the understanding of the 4-dimensional differential topology, since new smooth invariants were defined by the topology of their moduli space of solutions (moduli gauge group). In the same article, Witten introduced a variational formulation for the equations and showed that its stable critical points turn out to be exactly the $\mathscr{S}^{\mathscr{W}} \mathcal{W}_{\alpha}$-monopoles. The variational aspects of the $\mathscr{S}^{\mathscr{W}} \mathcal{W}_{\alpha}$-equations were first explored in [3], where they proved that the functional satisfies the Palais-Smale condition and the solutions of the Euler-Lagrange (2nd-order) equations share the same important analytical properties as the $\mathscr{S}^{a} W_{\alpha}$-monopoles. Therefore, it is natural to ask if the equations fit into a Morse-Bott-Smale theory, where the lower number of critical points 
is the Betti number of the configuration space. The topology of the configuration space was described in [1]. Besides, if the SW-theory is a Morse theory, another natural question is to argue about the existence of a Morse-Smale-Witten complex, as in [6]. In the last question, the $\mathscr{S}^{q} W_{\alpha}$-equations on manifolds endowed with tubular ends or boundary also demand attention. The analogy of the $\mathscr{S}^{\mathscr{W}} \mathcal{W}_{\alpha}$-equation's variational formulation, with the variational principle of the Ginzburg-Landau equation in superconductivity, further motivates the present study.

1.1. $\operatorname{Spin}^{c}$ structure. The space of $\operatorname{Spin}^{c}$ structures on $X$ is identified with

$$
\operatorname{Spin}^{c}(X)=\left\{\alpha+\beta \in H^{2}(X, \mathbb{Z}) \oplus H^{1}\left(X, \mathbb{Z}_{2}\right) \mid w_{2}(X)=\alpha(\bmod 2)\right\}
$$

For each $\alpha \in \operatorname{Spin}^{c}(X)$, there is a representation $\rho_{\alpha}: \mathrm{SO}_{4} \rightarrow \mathbb{C} l_{4}$, induced by a $\operatorname{Spin}^{c}$ representation, and a pair of vector bundles $\left(\mathscr{S}_{\alpha}^{+}, \mathscr{L}_{\alpha}\right)$ over $X$ (see [4]). Let $P_{\mathrm{SO}_{4}}$ be the frame bundle of $X$, so

(i) $\mathscr{S}_{\alpha}=P_{\mathrm{SO}_{4}} \times_{\rho_{\alpha}} V=\mathscr{Y}_{\alpha}^{+} \oplus \mathscr{Y}_{\alpha}^{-}$. The bundle $\mathscr{S}_{\alpha}^{+}$is the positive complex spinors bundle (fibers are $\mathrm{Spin}_{4}^{c}$-modules isomorphic to $\mathbb{C}^{2}$ ),

(ii) $\mathscr{L}_{\alpha}=P_{\mathrm{SO}_{4}} \times_{\operatorname{det}(\alpha)} \mathbb{C}$. It is called the determinant line bundle associated to the Spin ${ }^{c}$-structure $\alpha \cdot\left(c_{1}\left(\mathscr{L}_{\alpha}\right)=\alpha\right)$.

Thus, for each $\alpha \in \operatorname{Spin}^{c}(X)$, we associate a pair of bundles

$$
\alpha \in \operatorname{Spin}^{c}(X) \rightsquigarrow\left(\mathscr{L}_{\alpha}, \mathscr{S}_{\alpha}^{+}\right)
$$

From now on, we considered on $X$ a Riemannian metric $g$ and on $\mathscr{Y}_{\alpha}$ a Hermitian structure $h$.

Let $P_{\alpha}$ be the $U_{1}$-principal bundle over $X$ obtained as the frame bundle of $\mathscr{L}_{\alpha}\left(c_{1}\left(P_{\alpha}\right)=\right.$ $\alpha)$. Also, we consider the adjoint bundles

$$
\operatorname{Ad}\left(U_{1}\right)=P_{U_{1}} \times_{\text {Ad }} U_{1}, \quad \operatorname{ad}\left(\mathfrak{u}_{1}\right)=P_{U_{1}} \times_{\mathrm{ad}} \mathfrak{u}_{1},
$$

where $\operatorname{Ad}\left(U_{1}\right)$ is a fiber bundle with fiber $U_{1}$, and $\operatorname{ad}\left(\mathfrak{u}_{1}\right)$ is a vector bundle with fiber isomorphic to the Lie algebra $\mathfrak{u}_{1}$.

1.2. The main theorem. Let $\mathscr{A}_{\alpha}$ be (formally) the space of connections (covariant derivative) on $\mathscr{L}_{\alpha}, \Gamma\left(\mathscr{Y}_{\alpha}^{+}\right)$the space of sections of $\mathscr{S}_{\alpha}^{+}$, and $\mathscr{G}_{\alpha}=\Gamma\left(\operatorname{Ad}\left(U_{1}\right)\right)$ the gauge group acting on $\mathscr{A}_{\alpha} \times \Gamma\left(\mathscr{S}_{\alpha}^{+}\right)$as follows:

$$
g \cdot(A, \phi)=\left(A+g^{-1} d g, g^{-1} \phi\right)
$$

$\mathscr{A}_{\alpha}$ is an affine space with vector space structure, after fixing an origin, isomorphic to the space $\Omega^{1}\left(\operatorname{ad}\left(\mathfrak{u}_{1}\right)\right)$ of ad( $\left.\mathfrak{u}_{1}\right)$-valued 1-forms. Once a connection $\nabla^{0} \in \mathscr{A}_{\alpha}$ is fixed, a bijection $\mathscr{A}_{\alpha} \leftrightarrow \Omega^{1}\left(\operatorname{ad}\left(\mathfrak{u}_{1}\right)\right)$ is exposed by $\nabla^{A} \leftrightarrow A$, where $\nabla^{A}=\nabla^{0}+A$. $\mathscr{G}_{\alpha}=\operatorname{Map}\left(X, U_{1}\right)$, since $\operatorname{Ad}\left(U_{1}\right) \simeq X \times U_{1}$. The curvature of a 1-connection form $A \in \Omega^{1}\left(\operatorname{ad}\left(\mathfrak{u}_{1}\right)\right)$ is the 2form $F_{A}=d A \in \Omega^{2}\left(\operatorname{ad}\left(\mathfrak{u}_{1}\right)\right)$. 
Definition 1.1. (1) The configuration space of the D-problem is

$$
\mathscr{C}_{\alpha}^{\mathscr{D}}=\left\{(A, \phi) \in \mathscr{A}_{\alpha} \times \Gamma\left(\mathscr{Y}_{\alpha}^{+}\right)|(A, \phi)| \stackrel{\text { gauge }}{\sim}\left(A_{0}, \phi_{0}\right)\right\},
$$

(2) the configuration space of the $\mathcal{N}$-problem is

$$
\mathscr{C}_{\alpha}^{\mathcal{N}}=\mathscr{A}_{\alpha} \times \Gamma\left(\mathscr{S}_{\alpha}^{+}\right)
$$

Although each boundary problem requires its own configuration space, the superscripts $\mathscr{D}$ and $\mathcal{N}$ will be used whenever the distinction is necessary, since most arguments work for both sort of problems. The gauge group $\mathscr{G}_{\alpha}$ action on each of the configuration spaces is given by (1.4).

The Dirichlet $(\mathscr{D})$ and Neumann $(\mathcal{N})$ boundary value problems associated to the $\mathscr{S}^{0} W_{\alpha}$-equations are the following: we consider $(\Theta, \sigma) \in \Omega^{1}\left(\operatorname{ad}\left(\mathfrak{u}_{1}\right)\right) \oplus \Gamma\left(\mathscr{Y}_{\alpha}^{+}\right)$and $\left(A_{0}, \phi_{0}\right)$ defined on the manifold $\partial X\left(A_{0}\right.$ is a connection on $\left.\mathscr{L}_{\alpha}\right|_{\partial X}, \phi_{0}$ is a section of $\left.\Gamma\left(\left.\mathscr{Y}_{\alpha}^{+}\right|_{\partial X}\right)\right)$. In this way, find $(A, \phi) \in \mathscr{C}_{\alpha}^{\mathscr{D}}$ satisfying $\mathscr{D}$ and $(A, \phi) \in \mathscr{C}_{\alpha}^{\mathcal{N}}$ satisfying $\mathcal{N}$, where

(1)

$$
\mathscr{D}=\left\{\begin{array}{l}
d^{*} F_{A}+4 \Phi^{*}\left(\nabla^{A} \phi\right)=\Theta, \\
\Delta_{A} \phi+\frac{\left(|\phi|^{2}+k_{g}\right)}{4} \phi=\sigma, \\
\left.(A, \phi)\right|_{\partial X} \stackrel{\text { gauge }}{\sim}\left(A_{0}, \phi_{0}\right),
\end{array} \quad \mathcal{N}=\left\{\begin{array}{l}
d^{*} F_{A}+4 \Phi^{*}\left(\nabla^{A} \phi\right)=\Theta, \\
\Delta_{A} \phi+\frac{\left(|\phi|^{2}+k_{g}\right)}{4} \phi=\sigma, \\
i^{*}\left(* F_{A}\right)=0, \quad \nabla_{\nu}^{A} \phi=0,
\end{array}\right.\right.
$$

(2) the operator $\Phi^{*}: \Omega^{1}\left(\mathscr{S}_{\alpha}^{+}\right) \rightarrow \Omega^{1}\left(\mathfrak{u}_{1}\right)$ is locally given by

$$
\Phi^{*}\left(\nabla^{A} \phi\right)=\frac{1}{2} \nabla^{A}\left(|\phi|^{2}\right)=\sum_{i}\left\langle\nabla_{i}^{A} \phi, \phi\right\rangle \eta_{i},
$$

and $\eta=\left\{\eta_{i}\right\}$ is an orthonormal frame in $\Omega^{1}\left(\operatorname{ad}\left(\mathfrak{u}_{1}\right)\right)$,

(3) $i^{*}\left(* F_{A}\right)=F_{4}$, where $F_{4}=\left(F_{14}, F_{24}, F_{34}, 0\right)$ is the local representation of the 4 th component (normal to $\partial X)$ of the 2 -form of curvature in the local chart $(x, U)$ of $X ; x(U)=\left\{x=\left(x_{1}, x_{2}, x_{3}, x_{4}\right) \in \mathbb{R}^{4} ;\|x\|<\epsilon, x_{4} \geq 0\right\}$, and $x(U \cap \partial X) \subset\{x \in$ $\left.x(U) \mid x_{4}=0\right\}$. Let $\left\{e_{1}, e_{2}, e_{3}, e_{4}\right\}$ be the canonical base of $\mathbb{R}^{4}$, so $v=-e_{4}$ is the normal vector field along $\partial X$.

Theorem 1.2 (main theorem). If the pair $(\Theta, \sigma) \in L^{k, 2} \oplus\left(L^{k, 2} \cap L^{\infty}\right)$ satisfies the $\mathscr{H}$ Condition 3.1, then the problems $\mathscr{D}$ and $\mathcal{N}$ admit a $C^{r}$-regular solution $(A, \phi)$, whenever $2<k$ and $r<k$.

\section{Basic set up}

2.1. Sobolev spaces. As a vector bundle $E$ over $(X, g)$ is endowed with a metric and a covariant derivative $\nabla$, we define the Sobolev norm of a section $\phi \in \Omega^{0}(E)$ as

$$
\|\phi\|_{L^{k, p}}=\sum_{|i|=0}^{k}\left(\int_{X}\left|\nabla^{i} \phi\right|^{p}\right)^{1 / p} .
$$


In this way, the $L^{k, p}$-Sobolev Spaces of sections of $E$ is defined as

$$
L^{k, p}(E)=\left\{\phi \in \Omega^{0}(E) \mid\|\phi\|_{L^{k, p}}<\infty\right\} .
$$

In our context, in which we fixed a connection $\nabla^{0}$ on $\mathscr{L}_{\alpha}$, a metric $g$ on $X$, and a Hermitian structure on $\mathscr{S}_{\alpha}$, the Sobolev spaces on which the basic setting is made are the following:

(i) $\mathscr{A}_{\alpha}=L^{1,2}\left(\Omega^{1}\left(\operatorname{ad}\left(\mathfrak{u}_{1}\right)\right)\right)$;

(ii) $\Gamma\left(\mathscr{Y}_{\alpha}^{+}\right)=L^{1,2}\left(\Omega^{0}\left(X, \mathscr{Y}_{\alpha}^{+}\right)\right)$;

(iii) $\mathscr{C}_{\alpha}=\mathscr{A}_{\alpha} \times \Gamma\left(\mathscr{S}_{\alpha}^{+}\right)$;

(iv) $\mathscr{G}_{\alpha}=L^{2,2}\left(X, U_{1}\right)=L^{2,2}\left(\operatorname{Map}\left(X, U_{1}\right)\right)$. ( $\mathscr{G}_{\alpha}$ is an $\infty$-dimensional Lie group with Lie algebra $\left.\mathfrak{g}=L^{1,2}\left(X, \mathfrak{u}_{1}\right)\right)$.

The above Sobolev spaces induce a Sobolev structure on $\mathscr{C}_{\alpha}^{\mathfrak{D}}$ and on $\mathscr{C}_{\alpha}^{\mathcal{N}}$. From now on, the configuration spaces will be denoted by $\mathscr{C}_{\alpha}$ by ignoring the superscripts, unless needed.

The most basic analytical results needed to achieve the main result is the gauge fixing lemma (see [7]) and the estimate (2.3), both extended by Marini [5] to manifolds with boundary.

Lemma 2.1 (gauge fixing lemma). Every connection $\widehat{A} \in \mathscr{A}_{\alpha}$ is gauge equivalent, by a gauge transformation $g \in \mathscr{G}_{\alpha}$ named Coulomb ( $\left.\mathfrak{C}\right)$ gauge, to a connection $A \in \mathscr{A}_{\alpha}$ satisfying

(1) $d_{\tau}^{* f} A_{\tau}=0$ on $\partial X$

(2) $d^{*} A=0$ on $X$,

(3) in the $\mathcal{N}$-problem, the connection $A$ satisfies $A_{\nu}=0(\nu \perp \partial X)$.

Corollary 2.2. Under the hypothesis of Lemma 2.1, there exists a constant $K>0$ such that the connection A, gauge equivalent to $\widehat{A}$ by the Coulomb gauge, satisfies the following estimates:

$$
\|A\|_{L^{1, p}} \leq K \cdot\left\|F_{A}\right\|_{L^{p}}
$$

Notation. $*_{f}$ is the Hodge operator in the flat metric and the index $\tau$ denotes tangential components.

2.2. Variational formulation. A global formulation for problems $\mathscr{D}$ and $\mathcal{N}$ is made using the Seiberg-Witten functional.

Definition 2.3. Let $\alpha \in \operatorname{Spin}^{c}(X)$. The Seiberg-Witten functional $\mathscr{S}^{\mathscr{W}} \mathcal{W}_{\alpha}: \mathscr{C}_{\alpha} \rightarrow \mathbb{R}$ is defined as

$$
\mathscr{S} \mathcal{W}_{\alpha}(A, \phi)=\int_{X}\left\{\frac{1}{4}\left|F_{A}\right|^{2}+\left|\nabla^{A} \phi\right|^{2}+\frac{1}{8}|\phi|^{4}+\frac{k_{g}}{4}|\phi|^{2}\right\} d v_{g}+\pi^{2} \alpha^{2}
$$

where $k_{g}=$ scalar curvature of $(X, g)$. 
Remark 2.4. The $\mathscr{G}_{\alpha}$-action on $\mathscr{C}_{\alpha}$ has the following properties:

(1) the $\mathscr{S}^{\alpha} W_{\alpha}$-functional is $\mathscr{G}_{\alpha}$-invariant,

(2) the $\mathscr{G}_{\alpha}$-action on $\mathscr{C}_{\alpha}$ induces on $T \mathscr{C}_{\alpha}$ a $\mathscr{G}_{\alpha}$-action as follows: let $(\Lambda, V) \in T_{(A, \phi)} \mathscr{C}_{\alpha}$ and $g \in \mathscr{G}_{\alpha}$,

$$
g \cdot(\Lambda, V)=\left(\Lambda, g^{-1} V\right) \in T_{g \cdot(A, \phi)} \mathscr{C}_{\alpha}
$$

Consequently, $d\left(\mathscr{S}^{q} W_{\alpha}\right)_{g \cdot(A, \phi)}(g \cdot(\Lambda, V))=d\left(\mathscr{S}^{q} \mathcal{W}_{\alpha}\right)_{(A, \phi)}(\Lambda, V)$.

The tangent bundle $T \mathscr{C}_{\alpha}$ decomposes as

$$
T^{\mathscr{b}_{\alpha}}=\Omega^{1}\left(\operatorname{ad}\left(\mathfrak{u}_{1}\right)\right) \oplus \Gamma\left(\mathscr{Y}_{\alpha}^{+}\right)
$$

In this way, the 1 -form $d \mathscr{S}^{\alpha} W_{\alpha} \in \Omega^{1}\left(\mathscr{C}_{\alpha}\right)$ admits a decomposition $d \mathscr{S}^{\alpha} W_{\alpha}=d_{1} \mathscr{S}^{q} W_{\alpha}+$ $d_{2} \mathscr{S}^{q} W_{\alpha}$, where

$$
\begin{gathered}
d_{1}\left(\mathscr{S}^{\mathcal{W}}\right)_{(A, \phi)}: \Omega^{1}\left(\operatorname{ad}\left(\mathfrak{u}_{1}\right)\right) \longrightarrow \mathbb{R}, \quad d_{1}\left(\mathscr{S}^{\alpha} W_{\alpha}\right)_{(A, \phi)} \cdot \Lambda=d\left(\mathscr{S}^{\alpha} W_{\alpha}\right)_{(A, \phi)} \cdot(\Lambda, 0), \\
d_{2}\left(\mathscr{S}^{\alpha} W_{\alpha}\right)_{(A, \phi)}: \Gamma\left(\mathscr{Y}_{\alpha}^{+}\right) \longrightarrow \mathbb{R}, \quad d_{2}\left(\mathscr{S}^{\alpha} W_{\alpha}\right)_{(A, \phi)} \cdot V=d\left(\mathscr{S}^{\alpha} W_{\alpha}\right)_{(A, \phi)} \cdot(0, V) .
\end{gathered}
$$

By performing the computations, we get

(1) for every $\Lambda \in \mathscr{A}_{\alpha}$,

$$
d_{1}\left(\mathscr{S}^{\mathscr{W}}\right)_{(A, \phi)} \cdot \Lambda=\frac{1}{4} \int_{X} \operatorname{Re}\left\{\left\langle F_{A}, d_{A} \Lambda\right\rangle+4\left\langle\nabla^{A}(\phi), \Phi(\Lambda)\right\rangle\right\} d x,
$$

where $\Phi: \Omega^{1}\left(\mathfrak{u}_{1}\right) \rightarrow \Omega^{1}\left(\mathscr{Y}_{\alpha}^{+}\right)$is the linear operator $\Phi(\Lambda)=\Lambda(\phi)$, with dual defined in (1.8),

(2) for every $V \in \Gamma\left(\mathscr{Y}_{\alpha}^{+}\right)$,

$$
\left.d_{2}\left(\mathscr{S}^{\alpha}\right)_{\alpha}\right)_{(A, \phi)} \cdot V=\int_{X} \operatorname{Re}\left\{\left\langle\nabla^{A} \phi, \nabla^{A} V\right\rangle+\left\langle\frac{|\phi|^{2}+k_{g}}{4} \phi, V\right\rangle\right\} d x
$$

Therefore, by taking $\operatorname{supp}(\Lambda) \subset \operatorname{int}(X)$ and $\operatorname{supp}(V) \subset \operatorname{int}(X)$, we restrict to the interior of $X$, and so, the gradient of the $\mathscr{S}^{q} W_{\alpha}$-functional at $(A, \phi) \in \mathscr{C}_{\alpha}$ is

$$
\operatorname{grad}\left(\mathscr{S}^{\mathcal{W}}{ }_{\alpha}\right)(A, \phi)=\left(d_{A}^{*} F_{A}+4 \Phi^{*}\left(\nabla^{A} \phi\right), \triangle_{A} \phi+\frac{|\phi|^{2}+k_{g}}{4} \phi\right)
$$

It follows from the $\mathscr{G}_{\alpha}$-action on $T \mathscr{C}_{\alpha}$ that

$$
\operatorname{grad}\left(\mathscr{S}^{\alpha} W_{\alpha}\right)(g \cdot(A, \phi))=\left(d_{A}^{*} F_{A}+4 \Phi^{*}\left(\nabla^{A} \phi\right), g^{-1} \cdot\left(\triangle_{A} \phi+\frac{|\phi|^{2}+k_{g}}{4} \phi\right)\right)
$$

An important analytical aspect of the $\mathscr{S W}_{\alpha}$-functional is the coercivity lemma proved in $[3]$. 
Lemma 2.5 (coercivity). For each $(A, \phi) \in \mathscr{C}_{\alpha}$, there exist $g \in \mathscr{G}_{\alpha}$ and a constant $K_{C}^{(A, \phi)}>0$, where $K_{C}^{(A, \phi)}$ depends on $(X, g)$ and $\mathscr{S}^{\alpha} W_{\alpha}(A, \phi)$, such that

$$
\|g \cdot(A, \phi)\|_{L^{1,2}}<K_{C}^{(A, \phi)} .
$$

Proof (see [3, Lemma 2.3]). The gauge transform is the Coulomb one given in the Lemma 2.1 .

Considering the gauge invariance of the $\mathscr{S}^{0} W_{\alpha}$-theory, and the fact that the gauge group $\mathscr{G}_{\alpha}$ is an infinite-dimensional Lie group, we cannot hope to handle the problem in general. From now on, we need to restrict the problem to the space, named Coulomb subspace,

$$
\mathscr{C}_{\alpha}^{\mathfrak{C}}=\left\{(A, \phi) \in \mathscr{C}_{\alpha} ;\|(A, \phi)\|_{L^{1,2}}<K_{\mathfrak{C}}^{(A, \phi)}\right\} .
$$

The superscripts $\mathscr{D}$ and $\mathcal{N}$ have been omitted here for simplicity, although each one should be taken in account according to the problem. These choices of spaces come from the nature of the $\mathscr{G}_{\alpha}$ action on $\mathscr{C}_{\alpha}$, they are suggested by the gauge fixing lemma and the coercivity lemma (not shared by an actions in general).

\section{Existence of a solution}

3.1. Nonhomogeneous Palais-Smale condition - $\mathcal{H}$. In the variational formulation, the problems $\mathscr{D}$ and $\mathcal{N}(1.7)$ are written as

$$
\begin{aligned}
& (\mathscr{D})=\left\{\begin{array}{l}
\operatorname{grad}\left(\mathscr{S}^{\mathcal{W}} \mathcal{W}_{\alpha}\right)(A, \phi)=(\Theta, \sigma), \\
\left.(A, \phi)\right|_{\partial X} \underset{\sim}{\operatorname{gauge}}\left(A_{0}, \phi_{0}\right),
\end{array}\right. \\
& (\mathcal{N})=\left\{\begin{array}{l}
\operatorname{grad}\left(\mathscr{S}^{\mathcal{W}} W_{\alpha}\right)(A, \phi)=(\Theta, \sigma), \\
i^{*}\left(* F_{A}\right)=0, \quad \nabla_{n}^{A} \phi=0 .
\end{array}\right.
\end{aligned}
$$

The equations in (1.7) may not admit a solution for any pair $(\Theta, \sigma) \in \Omega^{1}\left(\operatorname{ad}\left(\mathfrak{u}_{1}\right)\right) \oplus$ $\Gamma\left(\mathscr{G}_{\alpha}^{+}\right)$. In finite dimension, if we consider a function $f: X \rightarrow \mathbb{R}$, the analogous question would be to find a point $p \in X$ such that, for a fixed vector $u$, $\operatorname{grad}(f)(p)=u$. This question is more subtle if $f$ is invariant under a Lie group action on $X$. Therefore, we need a hypothesis about the pair $(\Theta, \sigma) \in \Omega^{1}\left(\operatorname{ad}\left(\mathfrak{u}_{1}\right)\right) \oplus \Gamma\left(\mathscr{S}_{\alpha}^{+}\right)$.

Condition $3.1(\mathscr{H})$. Let $(\Theta, \sigma) \in L^{1,2}\left(\Omega^{1}\left(\operatorname{ad}\left(\mathfrak{u}_{1}\right)\right)\right) \oplus\left(L^{1,2}\left(\Gamma\left(\mathscr{Y}_{\alpha}^{+}\right)\right) \cap L^{\infty}\left(\Gamma\left(\mathscr{Y}_{\alpha}^{+}\right)\right)\right)$be a pair such that there exists a sequence $\left\{\left(A_{n}, \phi_{n}\right)\right\}_{n \in \mathbb{Z}} \subset \mathscr{C}_{\alpha}^{\mathfrak{C}}(2.13)$ with the following properties:

(1) $\left\{\left(A_{n}, \phi_{n}\right)\right\}_{n \in \mathbb{Z}} \subset L^{1,2}\left(\mathscr{A}_{\alpha}\right) \times\left(L^{1,2}\left(\Gamma\left(\mathscr{Y}_{\alpha}^{+}\right)\right) \cup L^{\infty}\left(\Gamma\left(\mathscr{Y}_{\alpha}^{+}\right)\right)\right)$and there exists a constant $c_{\infty}>0$ such that, for all $n \in \mathbb{Z},\left\|\phi_{n}\right\|_{\infty}<c_{\infty}$,

(2) there exists $c \in \mathbb{R}$ such that, for all $n \in \mathbb{Z}, \mathscr{S}^{\top} W_{\alpha}\left(A_{n}, \phi_{n}\right)<c$,

(3) the sequence $\left\{d\left(\mathscr{S}^{\alpha} W_{\alpha}\right)_{\left(A_{n}, \phi_{n}\right)}\right\}_{n \in \mathbb{Z}} \subset\left(L^{1,2}\left(\Omega^{1}\left(\operatorname{ad}\left(\mathfrak{u}_{1}\right)\right)\right) \oplus L^{1,2}\left(\Gamma\left(\mathscr{S}_{\alpha}^{+}\right)\right)\right)^{*}$, of linear functionals, converges weakly to

$$
L_{\Theta}+L_{\sigma}: T \mathscr{C}_{\alpha} \longrightarrow \mathbb{R}
$$


where

$$
L_{\Theta}(\Lambda)=\int_{X}\langle\Theta, \Lambda\rangle, \quad L_{\sigma}(V)=\int_{X}\langle\sigma, V\rangle
$$

3.2. Strong convergence of $\left\{\left(A_{n}, \phi_{n}\right)\right\}_{n \in \mathbb{Z}}$ in $L^{1,2}$. As a consequence of Lemma 2.5, the sequence $\left\{\left(A_{n}, \phi_{n}\right)\right\}_{n \in \mathbb{Z}}$ given by the $\mathcal{H}$-condition converges to a pair $(A, \phi)$;

(1) weakly in $\mathscr{C}_{\alpha}$,

(2) weakly in $L^{4}\left(\mathscr{A}_{\alpha} \times \Gamma\left(\mathscr{Y}_{\alpha}^{+}\right)\right)$,

(3) strongly in $L^{p}\left(\mathscr{A}_{\alpha} \times \Gamma\left(\mathscr{Y}_{\alpha}^{+}\right)\right)$, for every $p<4$.

Remark 3.2. Let $\left\{A_{n}\right\}_{n \in \mathbb{N}} \subset L^{2}$ be a converging sequence in $L^{2}$ satisfying $d^{*} A_{n}=0$, for all $n \in \mathbb{N}$, and let $A=\lim _{n \rightarrow \infty} A_{n} \in L^{2}$. So, $d^{*} A=0$, once

$$
\left|\left\langle d^{*} A, \rho\right\rangle\right| \leq\left|A-A_{n}\right|_{L^{2}} \cdot|d \rho|_{L^{2}}
$$

for all $\rho \in \Omega^{0}\left(\operatorname{ad}\left(\mathfrak{u}_{1}\right)\right)$.

Theorem 3.3. The limit $(A, \phi) \in L^{2}\left(\mathscr{A}_{\alpha} \times \Gamma\left(\mathscr{S}_{\alpha}^{+}\right)\right)$, obtained as a limit of the sequence $\left\{\left(A_{n}, \phi_{n}\right)\right\}_{n \in \mathbb{Z}}$, is a weak solution of (1.7).

Proof. The proof goes along the same lines as in the 2 nd step in the proof of the compactness theorem in [3].

(1) For every $\Lambda \in \mathscr{A}_{\alpha}$,

$$
\begin{aligned}
\left.d_{1}\left(\mathscr{S}^{q}\right)_{\alpha}\right)_{\left(A_{n}, \phi_{n}\right)} \cdot \Lambda= & \frac{1}{4} \int_{X} \operatorname{Re}\left\{\left\langle F_{A_{n}}, d_{A_{n}} \Lambda\right\rangle+4\left\langle\nabla^{A_{n}}\left(\phi_{n}\right), \Phi(\Lambda)\right\rangle\right\} d x \\
& +\int_{\partial X} \operatorname{Re}\left\{\Lambda \wedge * F_{A_{n}}\right\}
\end{aligned}
$$

where

(a) $\Phi: \Omega^{1}\left(\mathfrak{u}_{1}\right) \rightarrow \Omega^{1}\left(\mathscr{Y}_{\alpha}^{+}\right)$is the linear operator $\Phi(\Lambda)=\Lambda(\phi)$; its dual is defined in (1.8). Assuming $\phi \in L^{\infty}$ (Lemma 3.4), it follows that

$$
\lim _{n \rightarrow \infty} d_{1}\left(\mathscr{P}^{q} W_{\alpha}\right)_{\left(A_{n}, \phi_{n}\right)} \cdot \Lambda=d_{1}\left(\mathscr{S}^{q} W_{\alpha}\right)_{(A, \phi)} \cdot \Lambda
$$

Therefore, $d_{1}\left(\mathscr{S}^{q} W_{\alpha}\right)_{(A, \phi)} \cdot \Lambda=\int_{X}\langle\Theta, \Lambda\rangle$,

(b) $\Lambda \wedge * F_{A}=-\left\langle\Lambda, F_{4}\right\rangle d x_{1} \wedge d x_{2} \wedge d x_{3}$. Since the above equation is true for all $\Lambda$, let $\operatorname{supp}(\Lambda) \subset \partial X$, so $F_{4}=0\left(\Rightarrow i^{*}\left(* F_{A}\right)=0\right)$.

(2) For every $V \in \Gamma\left(\mathscr{S}_{\alpha}^{+}\right)$,

$$
\begin{aligned}
d_{2}\left(\mathscr{S}^{q} W_{\alpha}\right)_{\left(A_{n}, \phi_{n}\right)} \cdot V= & \int_{X} \operatorname{Re}\left\{\left\langle\nabla^{A_{n}} \phi_{n}, \nabla^{A_{n}} V\right\rangle+\left\langle\frac{\left|\phi_{n}\right|^{2}+k_{g}}{4} \phi_{n}, V\right\rangle\right\} d x \\
& +\int_{\partial X} \operatorname{Re}\left\{\left\langle\nabla_{\nu}^{A_{n}} \phi_{n}, V\right\rangle\right\} .
\end{aligned}
$$


Analogously, it follows that $(A, \phi)$ is a weak solution of the equation

$$
d_{2}\left(\mathscr{S} W_{\alpha}\right)_{(A, \phi)} \cdot V=\int_{X}\langle\sigma, V\rangle
$$

So, in the $\mathcal{N}$-problem, $\nabla_{\nu}^{A} \phi=0$.

In order to pursue the strong $L^{1,2}$-convergence for the sequence $\left\{\left(A_{n}, \phi_{n}\right)\right\}_{n \in \mathbb{Z}}$, we obtain in the following an upper bound for $\|\phi\|_{L^{\infty}}$, whenever $(A, \phi)$ is a weak solution.

Lemma 3.4. Let $(A, \phi)$ be a solution of either $\mathscr{D}$ or $\mathcal{N}$ in (1.7), so the following hold.

(1) If $\sigma=0$, then there exists a constant $k_{X, g}$, depending on the Riemannian metric on $X$, such that

$$
\|\phi\|_{\infty}<k_{X, g} \operatorname{vol}(X)
$$

(2) If $\sigma \neq 0$, then there exist constant $c_{1}=c_{1}(X, g)$ and $c_{2}=c_{2}(X, g)$ such that

$$
\|\phi\|_{L^{p}}<c_{1}+c_{2}\|\sigma\|_{L^{3 p}}^{3}
$$

In particular, if $\sigma \in L^{\infty}$, then $\phi \in L^{\infty}$.

Proof. Fix $r \in \mathbb{R}$ and suppose that there is a ball $B_{r^{-1}}\left(x_{0}\right)$, around the point $x_{0} \in X$, such that

$$
|\phi(x)|>r, \quad \forall x \in B_{r^{-1}}\left(x_{0}\right) .
$$

Define

$$
\eta= \begin{cases}\left(1-\frac{r}{|\phi|}\right) \phi & \text { if } x \in B_{r^{-1}}\left(x_{0}\right) \\ 0 & \text { if } x \in X-B_{r^{-1}}\left(x_{0}\right) .\end{cases}
$$

So,

$$
\begin{gathered}
|\eta| \leq|\phi|, \\
\nabla \eta=r \frac{\langle\phi, \nabla \phi\rangle}{|\phi|^{3}} \phi+\left(1-\frac{r}{|\phi|}\right) \nabla \phi \\
\Longrightarrow|\nabla \eta|^{2}=r^{2} \frac{\langle\phi, \nabla \phi\rangle^{2}}{|\phi|^{4}}+2 r\left(1-\frac{r}{|\phi|}\right) \frac{\langle\phi, \nabla \phi\rangle^{2}}{|\phi|^{3}}+\left(1-\frac{r}{|\phi|}\right)^{2}|\nabla \phi|^{2} \\
\Longrightarrow|\nabla \eta|^{2}<r^{2} \frac{|\nabla \phi|^{2}}{|\phi|^{2}}+2 r\left(1-\frac{r}{|\phi|}\right) \frac{|\nabla \phi|^{2}}{|\phi|}+\left(1-\frac{r}{|\phi|}\right)^{2}|\nabla \phi|^{2} .
\end{gathered}
$$


Since $r<|\phi|$,

$$
|\nabla \eta|^{2}<4|\nabla \phi|^{2}
$$

Hence, by (3.13) and (3.14), $\eta \in L^{1,2}$. The directional derivative of $\mathscr{S}^{\mathscr{W}} \mathcal{W}_{\alpha}$ in direction $\eta$ is given by

$$
d\left(\mathscr{S}^{\alpha} W_{\alpha}\right)_{(A, \phi)}(0, \eta)=\int_{X}\left[\left\langle\nabla^{A} \phi, \nabla^{A} \eta\right\rangle+\frac{|\phi|^{2}+k_{g}}{4}|\phi|(|\phi|-r)\right] .
$$

By (2.9),

$$
\int_{X}\left[\left\langle\nabla^{A} \phi, \nabla^{A} \eta\right\rangle+\frac{|\phi|^{2}+k_{g}}{4}|\phi|(|\phi|-r)\right]=\int_{X}\left\langle\sigma,\left(1-\frac{r}{|\phi|}\right) \phi\right\rangle .
$$

However,

$$
\int_{X}\left\langle\nabla^{A} \phi, \nabla^{A} \eta\right\rangle=\int_{X}\left[r \frac{\left\langle\phi, \nabla^{A} \phi\right\rangle^{2}}{|\phi|^{3}}+\left(1-\frac{r}{|\phi|}\right)|\nabla \phi|^{2}\right]>0 .
$$

So,

$$
\int_{X} \frac{|\phi|^{2}+k_{g}}{4}|\phi|(|\phi|-r)<\int_{X}\left\langle\sigma,\left(1-\frac{r}{|\phi|}\right) \phi\right\rangle<\int_{X}|\sigma|(|\phi|-r) .
$$

Hence,

$$
\int_{X}(|\phi|-r)\left(\frac{|\phi|^{2}+k_{g}}{4}|\phi|-|\sigma|\right)<0
$$

Since $r<|\phi(x)|$, whenever $x \in B_{r^{-1}}\left(x_{0}\right)$, it follows that

$$
\left(|\phi|^{2}+k_{g}\right)|\phi|<4|\sigma|, \quad \text { a.e. in } B_{r^{-1}}\left(x_{0}\right) \text {. }
$$

There are two cases to be analysed independently.

(1) $\sigma=0$. In this case, we get

$$
\left(|\phi|^{2}+k_{g}\right)|\phi|<0, \quad \text { a.e. }
$$

The scalar curvature plays a central role here: if $k_{g} \geq 0$, then $\phi=0$; otherwise,

$$
|\phi| \leq \max \left\{0,\left(-k_{g}\right)^{1 / 2}\right\}
$$


82 Boundary value problems for the $\mathscr{S}^{\mathscr{W}} \mathcal{W}_{\alpha}$-equations

Since $X$ is compact, we let $k_{X, g}=\max _{x \in X}\left\{0,\left[-k_{g}(x)\right]^{1 / 2}\right\}$, and so,

$$
\|\phi\|_{\infty}<k_{X, g} \operatorname{vol}(X)
$$

(2) Let $\sigma \neq 0$. The inequality (3.20) implies that

$$
|\phi|^{3}+k_{g}|\phi|-4|\sigma|<0, \quad \text { a.e. }
$$

Consider the polynomial

$$
Q_{\sigma(x)}(w)=w^{3}+k_{g} w-4|\sigma(x)|
$$

An estimate for $|\phi|$ is obtained by estimating the largest real number $w$ satisfying $Q_{\sigma(x)}(w)$ $<0 . Q_{\sigma(x)}$ being monic implies that $\lim _{w \rightarrow \infty} Q_{\sigma(x)}(w)=+\infty$. So, either $Q_{\sigma(x)}>0$, whenever $w>0$, or there exists a root $\rho \in(0, \infty)$. The first case would imply that

$$
Q_{\sigma(x)}(|\phi(x)|)>0, \quad \text { a.e., }
$$

contradicting (3.20). By the same argument, there exists a root $\rho \in(0, \infty)$ such that $Q_{\sigma(x)}(w)$ changes its sign in a neighborhood of $\rho$. Let $\rho$ be the largest root in $(0, \infty)$ with this property. By the Corollary A.2, there exist constants $c_{1}=c_{1}(X, g)$ and $c_{2}$ such that

$$
|\rho|<c_{1}+c_{2}|\sigma(x)|^{3}
$$

Consequently,

$$
|\phi(x)|<c_{1}+c_{2}|\sigma(x)|^{3}, \quad \text { a.e. in } B_{r^{-1}}\left(x_{0}\right)
$$

and

$$
\|\phi\|_{L^{p}}<C_{1}+C_{2}\|\sigma\|_{L^{3 p}}^{3} \quad \text { restricted to } B_{r^{-1}}\left(x_{0}\right),
$$

where $C_{1}, C_{2}$ are constants depending on $\operatorname{vol}\left(B_{r^{-1}}\left(x_{0}\right)\right)$. The inequality (3.29) can be extended over $X$ by using a $C^{\infty}$ partition of unity. Moreover, if $\sigma \in L^{\infty}$, then

$$
\|\phi\|_{\infty}<C_{1}+C_{2}\|\sigma\|_{\infty}^{3}
$$

where $C_{1}, C_{2}$ are constants depending on $\operatorname{vol}(X)$.

A sort of concentration lemma, proved in [3], can be extended as follows.

Lemma 3.5. Let $\left\{\left(A_{n}, \phi_{n}\right)\right\}_{n \in \mathbb{Z}}$ be the sequence given by the H-Condition 3.1. Then,

$$
\lim _{n \rightarrow \infty} \int_{X}\left\langle\Phi^{*}\left(\nabla^{A_{n}} \phi_{n}\right), A_{n}-A\right\rangle=0
$$


Proof. By (1.8),

$$
\begin{aligned}
& \lim _{n \rightarrow \infty} \int_{X}\left\langle\Phi^{*}\right.\left.\left(\nabla^{A_{n}} \phi_{n}\right), A_{n}-A\right\rangle=\lim _{n \rightarrow \infty} \int_{X}\left\langle\nabla_{i}^{A_{n}} \phi_{n}, \phi_{n}\right\rangle \cdot\left\langle\eta_{i}, A_{n}-A\right\rangle, \\
& \lim _{n \rightarrow \infty} \int_{X}\left\langle\nabla_{i}^{A_{n}} \phi_{n}, \phi_{n}\right\rangle \cdot\left\langle\eta_{i}, A_{n}-A\right\rangle \\
& \quad \leq \lim _{n \rightarrow \infty} \int_{X}\left|\left\langle\nabla_{i}^{A_{n}} \phi_{n}, \phi_{n}\right\rangle\right|^{2} \cdot \int_{X}\left|\left\langle\eta_{i}, A_{n}-A\right\rangle\right|^{2} \\
& \quad \leq \lim _{n \rightarrow \infty}\left[\int_{X}\left|\nabla_{i}^{A_{n}} \phi_{n}\right|^{2} \cdot\left|\phi_{n}\right|^{2}\right] \cdot \int_{X}\left|A_{n}-A\right|^{2} \\
& \quad \leq \lim _{n \rightarrow \infty} c_{\infty} \cdot\left[\int_{X}\left|\nabla_{i}^{A_{n}} \phi_{n}\right|^{2}\right] \cdot\left\|A_{n}-A\right\|_{L^{2}}^{2} \\
& \quad \leq \lim _{n \rightarrow \infty} c_{\infty} \cdot\left\|\phi_{n}\right\|_{L^{1,2}}^{2} \cdot\left\|A_{n}-A\right\|_{L^{2}}^{2}=0 .
\end{aligned}
$$

Theorem 3.6. Let $(\Theta, \sigma)$ be a pair satisfying the H-Condition 3.1. Then, the sequence $\left\{\left(A_{n}, \phi_{n}\right)\right\}_{n \in \mathbb{Z}}$, given by Condition 3.1, converges strongly to $(A, \phi) \in \mathscr{C}_{\alpha}$.

Proof. From Theorem 3.3, $\left\{\left(A_{n}, \phi_{n}\right)\right\}_{n \in \mathbb{Z}}$ converges weakly in $L^{1,2}$ to $(A, \phi) \in \mathscr{C}_{\alpha}$. The proof is splitted into 2 parts.

(1) $\lim _{n \rightarrow \infty}\left\|A_{n}-A\right\|_{L^{1,2}}=0$. Let $d^{*}: \Omega^{1}\left(\operatorname{ad}\left(\mathfrak{u}_{1}\right)\right) \rightarrow \Omega^{0}\left(\operatorname{ad}\left(\mathfrak{u}_{1}\right)\right)$. The operator $d:$ $\operatorname{ker}\left(d^{*}\right) \rightarrow \Omega^{2}\left(\operatorname{ad}\left(\mathfrak{u}_{1}\right)\right)$ being elliptic implies, by the fundamental elliptic estimate, that

$$
\left\|A_{n}-A\right\|_{L^{1,2}} \leq c|| d\left(A_{n}-A\right)\left\|_{L^{2}}+\right\| A_{n}-A \|_{L^{2}}
$$

The first term in the right-hand side is controlled as follows:

$$
\begin{aligned}
\left\|d A_{n}-d A\right\|_{L^{2}}^{2}= & \int_{X}\left\langle d\left(A_{n}-A\right), d\left(A_{n}-A\right)\right\rangle \\
= & \int_{X}\left\langle d A_{n}, d\left(A_{n}-A\right)\right\rangle-\int_{X}\left\langle d A, d\left(A_{n}-A\right)\right\rangle \\
= & \int_{X}\left\langle d^{*} F_{A_{n}}, A_{n}-A\right\rangle-\int_{X}\left\langle d^{*} F_{A}, A_{n}-A\right\rangle \\
= & d\left(\mathscr{S} W_{\alpha}\right)_{\left(A_{n}, \phi_{n}\right)}\left(A_{n}-A\right)-4 \int_{X}\left\langle\Phi^{*}\left(\nabla^{A_{n}} \phi_{n}\right), A_{n}-A\right\rangle \\
& \left.-d\left(\mathscr{S}^{\mathcal{W}}\right)_{\alpha}\right)_{(A, \phi)}\left(A_{n}-A\right)-4 \int_{X}\left\langle\Phi^{*}\left(\nabla^{A} \phi\right), A_{n}-A\right\rangle+o(1) \\
= & -4\left\{\int_{X}\left\langle\Phi^{*}\left(\nabla^{A_{n}} \phi_{n}\right), A_{n}-A\right\rangle+\int_{X}\left\langle\Phi^{*}\left(\nabla^{A} \phi\right), A_{n}-A\right\rangle\right\} \\
& +o(1), \quad \lim _{n \rightarrow \infty} o(1)=0 .
\end{aligned}
$$

Thus, it follows from Lemma 3.5 that $\lim _{n \rightarrow \infty}\left\|A_{n}-A\right\|_{L^{1,2}}=0$, and consequently, $A_{n} \rightarrow A$ strongly in $L^{4}$. 
84 Boundary value problems for the $\mathscr{S}^{\mathscr{W}} \mathrm{W}_{\alpha}$-equations

(2) $\lim _{n \rightarrow \infty}\left\|\phi_{n}-\phi\right\|_{L^{1,2}}=0$.

(1)

(2)

$$
\left\|\nabla^{0} \phi_{n}-\nabla^{0} \phi\right\|_{L^{2}}^{2}=\overbrace{\int_{X}\left\langle\nabla^{0} \phi_{n}, \nabla^{0}\left(\phi_{n}-\phi\right)\right\rangle}-\overbrace{\int_{X}\left\langle\nabla^{0} \phi, \nabla^{0}\left(\phi_{n}-\phi\right)\right\rangle} .
$$

The term (1) leads to

$$
\begin{aligned}
& \int_{X}\left\langle\nabla^{0} \phi_{n}, \nabla^{0}\left(\phi_{n}-\phi\right)\right\rangle \\
&=\int_{X}\left\langle\left(\nabla^{A_{n}}-A_{n}\right) \phi_{n},\left(\nabla^{A_{n}}-A_{n}\right)\left(\phi_{n}-\phi\right)\right\rangle \\
&=\int_{X}\left\langle\nabla^{A_{n}} \phi_{n}, \nabla^{A_{n}}\left(\phi_{n}-\phi\right)\right\rangle-\int_{X}\left\langle\nabla^{A_{n}} \phi_{n}, A_{n}\left(\phi_{n}-\phi\right)\right\rangle \\
&-\int_{X}\left\langle A_{n} \phi_{n}, \nabla^{A_{n}}\left(\phi_{n}-\phi\right)\right\rangle+\int_{X}\left\langle A_{n} \phi_{n}, A_{n}\left(\phi_{n}-\phi\right)\right\rangle \\
&= d(\overbrace{}^{\left.S^{2} W_{\alpha}\right)}\left(A_{n}, \phi_{n}\right)\left(\phi_{n}-\phi\right)-\int_{X} \frac{\left|\phi_{n}\right|^{2}+k_{g}}{4}\left\langle\phi_{n}, \phi_{n}-\phi\right\rangle \\
&-\overbrace{\int_{X}\left\langle\nabla^{A_{n}} \phi_{n}, A_{n}\left(\phi_{n}-\phi\right)\right\rangle}^{(13)}-\overbrace{\int_{X}\left\langle A_{n} \phi_{n}, \nabla^{A_{n}}\left(\phi_{n}-\phi\right)\right\rangle}^{(14)} \\
&+\overbrace{\int_{X}\left\langle A_{n} \phi_{n}, A_{n}\left(\phi_{n}-\phi\right)\right\rangle} .
\end{aligned}
$$

The term (2) in (3.35) leads to similar terms named (21), (22), (23), and (24). We analyze each one of the above-obtained overbraced terms.

(a) Terms (11) and (21):

$$
\begin{aligned}
& d\left(\mathscr{S}^{q} \mathcal{W}_{\alpha}\right)_{\left(A_{n}, \phi_{n}\right)}\left(\phi_{n}-\phi\right)-\int_{X} \frac{\left|\phi_{n}\right|^{2}+k_{g}}{4}\left\langle\phi_{n}, \phi_{n}-\phi\right\rangle+o(1) \\
& \quad=\left\langle\sigma, \phi_{n}-\phi\right\rangle-\int_{X} \frac{\left|\phi_{n}\right|^{2}+k_{g}}{4}\left|\phi_{n}-\phi\right|^{2}-\int_{X} \frac{\left|\phi_{n}\right|^{2}+k_{g}}{4}\left\langle\phi, \phi_{n}-\phi\right\rangle+o(1) \\
& \leq\left\langle\sigma, \phi_{n}-\phi\right\rangle-\int_{X} \frac{\left|\phi_{n}\right|^{2}+k_{g}}{4}\left\langle\phi, \phi_{n}-\phi\right\rangle+o(1) \\
& \leq\|\sigma\|_{L^{2}}^{2} \cdot\left\|\phi_{n}-\phi\right\|_{L^{2}}^{2}+\left\|\frac{\left|\phi_{n}\right|^{2}+k_{g}}{4}\right\|_{L^{2}}^{2} \cdot\|\phi\|_{\infty} \cdot\left\|\phi_{n}-\phi\right\|_{L^{2}}^{2}+o(1),
\end{aligned}
$$

where $\lim _{n \rightarrow \infty} o(1)=0$. By the similarity between (11) and (21), we conclude the boundedness of term (22). 
(b) Terms (12) and (22):

(i) term (12):

$$
\begin{aligned}
\int_{X}\left\langle\nabla^{A_{n}} \phi_{n}, A_{n}\left(\phi_{n}-\phi\right)\right\rangle \\
=\int_{X}\left\langle\nabla^{A_{n}} \phi_{n},\left(A_{n}-A\right)\left(\phi_{n}-\phi\right)\right\rangle+\int_{X}\left\langle\nabla^{A_{n}} \phi_{n}, A\left(\phi_{n}-\phi\right)\right\rangle \\
\leq \int_{X}\left|\nabla^{A_{n}} \phi_{n}\right|^{2} \cdot \int_{X}\left|A_{n}-A\right|^{4} \cdot \int_{X}\left|\phi_{n}-\phi\right|^{4} \\
\quad+\int\left|\nabla^{A_{n}} \phi_{n}\right|^{2} \cdot \int_{X}\left|A\left(\phi_{n}-\phi\right)\right|^{2},
\end{aligned}
$$

(ii) term (22)

$$
\int_{X}\left\langle\nabla^{A} \phi, A\left(\phi_{n}-\phi\right)\right\rangle \leq \int_{X}\left|\nabla^{A} \phi\right|^{2} \cdot \int_{X}\left|A\left(\phi_{n}-\phi\right)\right|^{2} .
$$

The term $\int_{X}\left|\nabla^{A} \phi\right|^{2}$ is bounded by Proposition 4.1 and $A \in C^{0}$ by Theorem 4.4 .

(c) $\operatorname{Term}\{(13)-(23)\}$ :

$$
\begin{aligned}
\int_{X}\left\langle A_{n} \phi_{n}, \nabla^{A_{n}}\left(\phi_{n}-\phi\right)\right\rangle-\int_{X}\left\langle A \phi, \nabla^{A}\left(\phi_{n}-\phi\right)\right\rangle & \\
= & \int_{X}\left\langle\left(A_{n}-A\right) \phi_{n}, \nabla^{A_{n}}\left(\phi_{n}-\phi\right)\right\rangle+\overbrace{\int_{X}\left\langle A \phi_{n}, \nabla^{A_{n}}\left(\phi_{n}-\phi\right)\right\rangle}^{\text {(i) }} \\
& -\int_{X}\left\langle\left(A_{n}-A\right) \phi, \nabla^{A}\left(\phi_{n}-\phi\right)\right\rangle-\overbrace{\int_{X}\left\langle A_{n} \phi, \nabla^{A}\left(\phi_{n}-\phi\right)\right\rangle} .
\end{aligned}
$$

In each of the last two lines above, the first terms are bounded by $\left\|A_{n}-A\right\|_{L^{4}}$, while the term $\{(\mathrm{i})$-(ii) $\}$ can be written as

$$
\begin{aligned}
\int_{X}\langle(A & \left.\left.-A_{n}\right) \phi_{n}, \nabla^{A_{n}}\left(\phi_{n}-\phi\right)\right\rangle+\int_{X}\left\langle A_{n}\left(\phi_{n}-\phi\right), \nabla^{A_{n}}\left(\phi_{n}-\phi\right)\right\rangle \\
& +\int_{X}\left\langle A_{n} \phi,(\overbrace{\nabla^{A_{n}}-\nabla^{A}}^{\left(A_{n}-A\right)})\left(\phi_{n}-\phi\right)\right\rangle .
\end{aligned}
$$

So, it is also bounded by $\left\|A_{n}-A\right\|_{L^{4}}$.

(d) $\operatorname{Term}\{(14)-(24)\}$ :

$$
\begin{aligned}
\int_{X}\left\langle A_{n} \phi_{n}, A_{n}\left(\phi_{n}-\phi\right)\right\rangle-\int_{X}\left\langle A \phi, A\left(\phi_{n}-\phi\right)\right\rangle \\
=\int_{X}\left\langle A_{n} \phi_{n},\left(A_{n}-A\right)\left(\phi_{n}-\phi\right)\right\rangle+\int_{X}\left\langle\left(A_{n}-A\right) \phi_{n}, A\left(\phi_{n}-\phi\right)\right\rangle \\
\quad+\int\left|A\left(\phi_{n}-\phi\right)\right|^{2} .
\end{aligned}
$$

Since $A \in C^{0}$, it follows that $\lim _{n \rightarrow \infty}\left\|A\left(\phi_{n}-\phi\right)\right\|^{2}=0$. 


\section{Regularity of the solution $(A, \phi)$}

Let $\beta=\left\{e_{i} ; 1 \leq i \leq 4\right\}$ be an orthonormal frame fixed on $T X$ with the following properties; for all $i, j \in\{1,2,3,4\}$ :

(1) $\left[e_{i}, e_{j}\right]=0$,

(2) $\nabla_{e_{i}} e_{j}=0(\nabla=$ Levi-Civita connection on $X)$.

Let $\beta^{*}=\left\{d x_{1}, \ldots, d x_{n}\right\}$ be the dual frame induced on $\mathscr{S}_{\alpha}^{*}$. From the 2 nd property of the frame $\beta$, it follows that $\nabla_{e_{i}} d x^{j}=0$ for all $i, j \in\{1,2,3,4\}$. For the sake of simplicity, let $\nabla_{e_{i}}^{A}=\nabla_{i}^{A}$. Therefore, $\nabla^{A}: \Omega^{0}\left(\operatorname{ad}\left(\mathfrak{u}_{1}\right)\right) \rightarrow \Omega^{1}\left(\operatorname{ad}\left(\mathfrak{u}_{1}\right)\right)$ is given by

$$
\begin{gathered}
\nabla^{A} \phi=\sum_{l}\left(\nabla_{l}^{A} \phi\right) d x_{l} \Longrightarrow\left|\nabla^{A} \phi\right|^{2}=\sum_{l}\left|\nabla_{l}^{A} \phi\right|^{2} \\
\left(\nabla^{A}\right)^{2}=\sum_{k, l}\left(\nabla_{k}^{A} \nabla_{l}^{A} \phi\right) d x_{l} \wedge d x_{k} \Longrightarrow\left|\left(\nabla^{A}\right)^{2}\right|^{2}=\sum_{k, l}\left|\nabla_{k}^{A} \nabla_{l}^{A} \phi\right|^{2} .
\end{gathered}
$$

In this setting, the 2 form of curvature of the connection $A$ is given by

$$
\left(F_{A}\right)_{k l}=F_{k l}=\nabla_{l}^{A} \nabla_{k}^{A}-\nabla_{k}^{A} \nabla_{l}^{A}
$$

In order to compute the operator $\Delta_{A}=\left(\nabla^{A}\right)^{*} \nabla^{A}: \Omega^{0}\left(\mathscr{Y}_{\alpha}^{+}\right) \rightarrow \Omega^{0}\left(\mathscr{Y}_{\alpha}^{+}\right)$, let $*: \Omega^{i}\left(\mathscr{Y}_{\alpha}\right) \rightarrow$ $\Omega^{4-i}\left(\mathscr{S}_{\alpha}\right)$ be the Hodge operator and consider the identity

$$
\left(\nabla^{A}\right)^{*}=-* \nabla^{A} *: \Omega^{1}\left(\mathscr{Y}_{\alpha}^{+}\right) \longrightarrow \Omega^{0}\left(\mathscr{Y}_{\alpha}^{+}\right) .
$$

Hence,

$$
\Delta_{A} \phi=-\sum_{k} \nabla_{k}^{A} \nabla_{k}^{A} \phi
$$

In this way,

$$
\begin{aligned}
\left|\Delta_{A} \phi\right|^{2}= & \sum_{k, l}\left\langle\nabla_{k}^{A} \nabla_{k}^{A} \phi, \nabla_{l}^{A} \nabla_{l}^{A} \phi\right\rangle \\
= & \sum_{k, l}\left[\nabla_{k}^{A}\left(\left\langle\nabla_{k}^{A} \phi, \nabla_{l}^{A} \nabla_{l}^{A} \phi\right\rangle\right)-\left\langle\nabla_{k}^{A} \phi, \nabla_{k}^{A} \nabla_{l}^{A} \nabla_{l}^{A} \phi\right\rangle\right] \\
= & \sum_{k, l}\left[\nabla_{k}^{A}\left(\left\langle\nabla_{k}^{A} \phi, \nabla_{l}^{A} \nabla_{l}^{A} \phi\right\rangle\right)-\left\langle\nabla_{k}^{A} \phi, \nabla_{l}^{A} \nabla_{k}^{A} \nabla_{l}^{A} \phi\right\rangle-\left\langle\nabla_{k}^{A} \phi, F_{l k} \nabla_{l}^{A} \phi\right\rangle\right] \\
= & \sum_{k, l}\left[\nabla_{k}^{A}\left(\left\langle\nabla_{k}^{A} \phi, \nabla_{l}^{A} \nabla_{l}^{A} \phi\right\rangle\right)-\nabla_{l}^{A}\left(\left\langle\nabla_{k}^{A} \phi, \nabla_{k}^{A} \nabla_{l}^{A} \phi\right\rangle\right)\right] \\
& +\sum_{k, l}\left[\left\langle\nabla_{l}^{A} \nabla_{k}^{A} \phi, \nabla_{k}^{A} \nabla_{l}^{A} \phi\right\rangle+\left\langle\nabla_{k}^{A} \phi, F_{l k} \nabla_{l}^{A} \phi\right\rangle\right] \\
= & \sum_{k, l}\left[\nabla_{k}^{A}\left(\left\langle\nabla_{k}^{A} \phi, \nabla_{l}^{A} \nabla_{l}^{A} \phi\right\rangle\right)-\nabla_{l}^{A}\left(\left\langle\nabla_{k}^{A} \phi, \nabla_{k}^{A} \nabla_{l}^{A} \phi\right\rangle\right)\right]+\sum_{k, l}\left|\nabla_{k}^{A} \nabla_{l}^{A} \phi\right|^{2} \\
& +\sum_{k, l}\left[\left\langle F_{k l} \phi, \nabla_{k}^{A} \nabla_{l}^{A} \phi\right\rangle+\left\langle\nabla_{k}^{A} \phi, F_{k l} \nabla_{l}^{A} \phi\right\rangle\right]
\end{aligned}
$$


and so,

$$
\begin{aligned}
\left|\left(\nabla^{A}\right)^{2} \phi\right|^{2} \leq & \left|\Delta_{A} \phi\right|^{2}+\sum_{k, l}\left\{\left|\nabla_{k}^{A}\left(\left\langle\nabla_{k}^{A} \phi, \nabla_{l}^{A} \nabla_{l}^{A} \phi\right\rangle\right)\right|\right\}+\sum_{k, l}\left\{\left|\nabla_{l}^{A}\left(\left\langle\nabla_{k}^{A} \phi, \nabla_{k}^{A} \nabla_{l}^{A} \phi\right\rangle\right)\right|\right\} \\
& +\sum_{k, l}\left\{\left|\left\langle F_{k l} \phi, \nabla_{k}^{A} \phi \nabla_{l}^{A} \phi\right\rangle\right|\right\}+\sum_{k, l}\left\{\left|\left\langle\nabla_{k}^{A} \phi, F_{k l} \nabla_{l}^{A} \phi\right\rangle\right|\right\} .
\end{aligned}
$$

Now, by applying the inequalities

$$
\left(\sum_{i} a_{i}\right)^{r} \leq K_{r} \cdot \sum_{i}\left|a_{i}\right|^{r}, \quad \sqrt{\sum_{i=1}^{n} a_{i}} \leq \sum_{i=1}^{n} \sqrt{a_{i}}
$$

to (4.6), we get

$$
\begin{aligned}
\left|\left(\nabla^{A}\right)^{2} \phi\right|^{p} \leq & K_{p} \cdot\left|\Delta_{A} \phi\right|^{p}+K_{p} \cdot \sum_{k, l}\left\{\left|\nabla_{k}^{A}\left(\left\langle\nabla_{k}^{A} \phi, \nabla_{l}^{A} \nabla_{l}^{A} \phi\right\rangle\right)\right|^{p / 2}\right\} \\
& +K_{p} \sum_{k, l}\left\{\left|\nabla_{l}^{A}\left(\left\langle\nabla_{k}^{A} \phi, \nabla_{k}^{A} \nabla_{l}^{A} \phi\right\rangle\right)\right|^{p / 2}\right\} \\
& +\sum_{k, l}\left\{\left|\left\langle F_{k l} \phi, \nabla_{k}^{A} \phi \nabla_{l}^{A} \phi\right\rangle\right|^{p / 2}\right\}+\sum_{k, l}\left\{\left|\left\langle\nabla_{k}^{A} \phi, F_{k l} \nabla_{l}^{A} \phi\right\rangle\right|^{p / 2}\right\} .
\end{aligned}
$$

After integrating, it follows that

$$
\begin{aligned}
k_{1} \cdot\left\|\left(\nabla^{A}\right)^{2} \phi\right\|_{L^{p}}^{p} \leq & \left\|\Delta_{A} \phi\right\|_{L^{p}}^{p}+k_{2} \cdot\left\|\nabla^{A} \phi\right\|_{L^{p}}^{p}+k_{3} \cdot\left\|F_{A}(\phi)\right\|_{L^{p}}^{p} \\
& +k_{4} \cdot\left\|F_{A}\left(\nabla^{A} \phi\right)\right\|_{L^{p}}^{p}+k_{5} \cdot \sum_{k, l} \int_{x}\left\{\left|\nabla_{k}^{A}\left(\left\langle\nabla_{k}^{A} \phi, \nabla_{l}^{A} \nabla_{l}^{A} \phi\right\rangle\right)\right|^{p / 2}\right\} \\
& +k_{6} \sum_{k, l} \int_{X}\left\{\left|\nabla_{l}^{A}\left(\left\langle\nabla_{k}^{A} \phi, \nabla_{k}^{A} \nabla_{l}^{A} \phi\right\rangle\right)\right|^{p / 2}\right\} .
\end{aligned}
$$

The boundedness of the right-hand side of (4.9) results from the analysis of each term.

Proposition 4.1. Let $(A, \phi) \in \mathscr{C}_{\alpha}$ be a solution of equations in (1.7). If $\sigma \in L^{\infty}$, then

(1) $\nabla^{A} \phi \in L^{2}$,

(2) $\Delta_{A} \phi \in L^{2}$.

Proof. (1) $\nabla^{A} \phi \in L^{2}$ :

$$
\begin{aligned}
& \left\langle\Delta_{A} \phi, \phi\right\rangle+\left(\frac{|\phi|^{2}+k_{g}}{4}\right)|\phi|^{2}=\langle\sigma, \phi\rangle \\
& \Longrightarrow\left|\nabla^{A} \phi\right|^{2}+\left(\frac{|\phi|^{2}+k_{g}}{4}\right)|\phi|^{2}=\langle\sigma, \phi\rangle \leq \frac{1}{\epsilon^{2}}|\sigma|^{2}+\epsilon^{2}|\phi|^{2} .
\end{aligned}
$$

Therefore,

$$
\left|\nabla^{A} \phi\right|^{2}<\frac{1}{\epsilon^{2}}|\sigma|^{2}+\left(\epsilon^{2}-\frac{k_{g}}{4}\right)|\phi|^{2}-\frac{|\phi|^{4}}{4}
$$


From Lemma 3.4, there exists a polynomial $p$, with coefficients depending on $(X, g)$ and $\epsilon$, such that

$$
\left\|\nabla^{A} \phi\right\|_{L^{2}}^{2}<p\left(\|\sigma\|_{\infty}\right)
$$

So, $\nabla^{A} \phi \in L^{2}$.

(2) $\Delta_{A} \phi \in L^{2}$ :

$$
\left\langle\Delta_{A} \phi, \Delta_{A} \phi\right\rangle+\frac{|\phi|^{2}+k_{g}}{4}\left\langle\phi, \Delta_{A} \phi\right\rangle=\left\langle\sigma, \Delta_{A} \phi\right\rangle
$$

let $0<\epsilon<1$,

$$
\begin{gathered}
\left|\Delta_{A} \phi\right|^{2}+\frac{|\phi|^{2}+k_{g}}{4}\left|\nabla^{A} \phi\right|^{2}=\left\langle\sigma, \Delta_{A} \phi\right\rangle<\frac{1}{\epsilon^{2}}|\sigma|^{2}+\epsilon^{2}\left|\Delta_{A} \phi\right|^{2}, \\
\left(1-\epsilon^{2}\right)\left|\Delta_{A} \phi\right|^{2}+\frac{|\phi|^{2}+k_{g}}{4}\left|\nabla^{A} \phi\right|^{2}<\frac{1}{\epsilon^{2}}|\sigma|^{2} .
\end{gathered}
$$

By the boundedness of the term

$$
\int_{X}|\phi|^{2} \cdot\left|\nabla^{A} \phi\right|^{2}<\|\phi\|_{\infty}^{2} \cdot\left\|\nabla^{A} \phi\right\|_{L^{2}}^{2}
$$

one deduces the existence of a polynomial $q$, with coefficients depending on $\epsilon$ and $(X, g)$, such that

$$
\left\|\Delta_{A} \phi\right\|_{L^{2}}<q\left(\|\sigma\|_{\infty}\right)
$$

Proposition 4.2. Let $(A, \phi)$ be solutions of the $\mathscr{S}^{q} W_{\alpha}$-equations, where $(\Theta, \sigma) \in L^{1,2} \times\left(L^{1,2} \cap\right.$ $\left.L^{\infty}\right)$, then $F_{A} \in L^{q}$, for all $q<\infty$.

Proof. By (1.8), $\Phi^{*}\left(\nabla^{A} \phi\right)=(1 / 2) \nabla^{A}\left(|\phi|^{2}\right)$, and so,

$$
d^{*} F_{A}+4 \Phi^{*}\left(\nabla^{A} \phi\right)=\Theta \Longrightarrow\left\|d^{*} F_{A}\right\|_{L^{2}}^{2} \leq\|\phi\|_{L^{1,2}}^{2}+\|\Theta\|_{L^{2}}
$$

There are two cases to be analysed.

(1) $F_{A}$ is harmonic. Since the Laplacian defined on $\mathfrak{u}_{1}$-forms is an elliptic operator, the fundamental inequality for elliptic operators asserts that there exists a constant $C_{k}$ such that

$$
\left\|F_{A}\right\|_{L^{k+2,2}} \leq\left\|\Delta F_{A}\right\|_{L^{k, 2}}+C_{k}\left\|F_{A}\right\|_{L^{2}}
$$

Consequently, $F_{A}$ being harmonic implies, for all $k \in \mathbb{N}$, that

$$
\left\|F_{A}\right\|_{L^{k, 2}} \leq C_{k}\left\|F_{A}\right\|_{L^{2}} \Longrightarrow F_{A} \in C^{\infty} .
$$

(2) $F_{A}$ is not harmonic. In this case, since $\Theta \in L^{1,2}, \phi \in L^{\infty}$ and

$$
\Delta_{A} F_{A}=d\left(\left\langle\phi, \nabla^{A} \phi\right\rangle\right)+d \Theta=\left\langle\phi, F_{A}(\phi)\right\rangle+d \Theta,
$$


it follows that $F_{A} \in L^{2,2}$. Therefore, by the Sobolev embedding theorem, $F_{A} \in L^{q}$, for all $q<\infty$.

Proposition 4.3. Let $(A, \phi)$ be solutions of the $\mathscr{S}^{q} W_{\alpha}$-equations, where $(\Theta, \sigma) \in L^{1,2} \times\left(L^{1,2} \cap\right.$ $\left.L^{\infty}\right)$, then $\left(\nabla^{A}\right)^{2} \phi \in L^{p}$, for all $1<p<2$.

Proof. In (4.9), we must take care of the last terms.

(1) $F\left(\nabla^{A} \phi\right) \in L^{p}$, for all $1<p<2$. By Young's inequality,

$$
\left\|F\left(\nabla^{A} \phi\right)\right\|_{L^{p}} \leq\left\|F_{A}\right\|_{L^{2 p /(2-p)}} \cdot\left\|\nabla^{A} \phi\right\|_{L^{2}}
$$

(2) There is no contribution from the divergent terms, since

$$
\int_{x}\left\{\left|\nabla_{k}^{A}\left(\left\langle\nabla_{k}^{A} \phi, \nabla_{l}^{A} \nabla_{l}^{A} \phi\right\rangle\right)\right|^{p / 2}\right\} \leq[\operatorname{vol}(X)]^{(2-p) / p} \int_{x}\left\{\left|\nabla_{k}^{A}\left(\left\langle\nabla_{k}^{A} \phi, \nabla_{l}^{A} \nabla_{l}^{A} \phi\right\rangle\right)\right|\right\} .
$$

In the same way,

$$
\begin{aligned}
& \sum_{k, l} \int_{x}\left\{\left|\nabla_{k}^{A}\left(\left\langle\nabla_{k}^{A} \phi, \nabla_{l}^{A} \nabla_{l}^{A} \phi\right\rangle\right)\right|^{p / 2}\right\}=0, \\
& \sum_{k, l} \int_{X}\left\{\left|\nabla_{l}^{A}\left(\left\langle\nabla_{k}^{A} \phi, \nabla_{k}^{A} \nabla_{l}^{A} \phi\right\rangle\right)\right|^{p / 2}\right\}=0 .
\end{aligned}
$$

The estimates above applied to (4.9) implies that

$$
\begin{aligned}
\left\|\left(\nabla^{A}\right)^{2} \phi\right\|_{L^{p}} \leq & k_{1}\left\|\Delta_{A} \phi\right\|_{L^{p}}^{p}+k_{2}\left\|\nabla^{A} \phi\right\|_{L^{p}}^{p}+k_{3}\left\|\nabla^{A} \phi\right\|_{L^{p}}^{p} \\
& +k_{4}\left\|F_{A}(\phi)\right\|_{L^{p}}^{p}+k_{5}\left\|F_{A}\right\|_{L^{p /(2-p)}} \cdot\left\|\nabla^{A} \phi\right\|_{L^{p}}^{p} .
\end{aligned}
$$

Thus, $\phi \in L^{2, p}$, for all $1<p<2$. Considering that $\sigma \in L^{1,2}$, the bootstrap argument applied on (1.7) implies that $\phi \in L^{3, p}$, for every $k \geq 2$ and $1<p<2$. Hence, by Sobolev embedding theorem, $\phi \in C^{0}$.

TheOREM 4.4. Let $(A, \phi)$ be a solution of the $\mathscr{S}^{\alpha} W_{\alpha}$-equations, where $(\Theta, \sigma) \in$ $L^{k, 2}\left(\Omega^{1}\left(\operatorname{ad}\left(\mathfrak{u}_{1}\right)\right)\right) \oplus\left(L^{k, 2}\left(\Gamma\left(\mathscr{Y}_{\alpha}^{+}\right)\right) \cap L^{\infty}\left(\Gamma\left(\mathscr{Y}_{\alpha}^{+}\right)\right)\right)$, then $(A, \phi) \in L^{k+2, p} \times\left(L^{k+2,2} \cap L^{\infty}\right)$, for all $1<p<2$. Moreover, if $k>2$, then $(A, \phi) \in C^{r} \times C^{r}$, for all $r<k$.

Proof. (1) If $\Theta \in L^{k, 2}$, then by Proposition $4.2 F_{A} \in L^{k+1,2}$. Consequently, by Corollary 2.2, $A \in L^{k+2,2}$.

(2) The Sobolev class of $\phi$ is obtained by the bootstrap argument.

\section{Appendix}

\section{Estimates for solutions of 3 rd-degree equation}

Let $p, q \in \mathbb{R}$ and consider the equation

$$
x^{3}+p x+q=0
$$


90 Boundary value problems for the $\mathscr{S}^{q} \mathcal{W}_{\alpha}$-equations

Proposition A.1. The solutions of (A.1) are given in [2] by

$$
x_{1}=z_{1}+z_{2}, \quad x_{2}=z_{1}+\lambda z_{2}, \quad y_{3}=z_{1}+\lambda^{2} z_{2},
$$

where

$$
z_{1}=\sqrt[3]{-\frac{q}{2}+\sqrt[2]{D}}, \quad z_{2}=\sqrt[3]{-\frac{q}{2}-\sqrt[2]{D}}, \quad D=\frac{p^{3}}{27}+\frac{q^{2}}{4}
$$

and $\lambda \in \mathbb{C}$ satisfies $\lambda^{3}=1$.

Corollary A.2. Let $p$ and $q$ be negative real numbers. So, the solutions of (A.1) are estimated according to the following cases:

(1) $D \geq 0$ :

$$
\left|x_{i}\right| \leq \frac{8}{3}+\frac{1}{3}|q|+\frac{1}{12} q^{2}+\frac{1}{81} p^{3}
$$

(2) $D<0$ :

$$
\left|x_{i}\right| \leq 3+\frac{1}{6} q^{2}+\frac{1}{81}|p|^{3}
$$

Proof. Since

$$
\left|x_{i}\right| \leq\left|z_{1}\right|+\left|z_{2}\right|
$$

it is enough to estimate $\left|z_{1}\right|$ and $\left|z_{2}\right|$. The basics identities needed are the following: suppose $x \geq 0$, whence

$$
\sqrt[2]{x} \leq 1+\frac{1}{2} x, \quad \sqrt[3]{x} \leq 1+\frac{1}{3} x
$$

(1) $D \geq 0$. In this case, $z_{1}, z_{2} \in \mathbb{R}$ and

$$
\left|z_{1}\right|=\sqrt[3]{\left|-\frac{q}{2}+\sqrt[2]{D}\right|} \leq 1+\frac{1}{3}\left|-\frac{q}{2}+\sqrt[2]{D}\right| \leq \frac{4}{3}+\frac{1}{6}|q|+\frac{1}{6} D .
$$

Thus,

$$
\left|z_{1}\right| \leq \frac{4}{3}+\frac{1}{6}|q|+\frac{1}{24} q^{2}+\frac{1}{162} p^{3} .
$$

The same estimate can be obtained for $\left|z_{2}\right|$. Hence,

$$
\left|x_{i}\right| \leq \frac{8}{3}+\frac{1}{3}|q|+\frac{1}{12} q^{2}+\frac{1}{81} p^{3}
$$


(2) $D \leq 0$. In this case, $z_{1}, z_{2} \in \mathbb{C}-\mathbb{R}$. Since $D \in \mathbb{R}$, we can write $\sqrt[2]{D}=i \sqrt[2]{|D|}$ and

$$
z_{1}=\sqrt[3]{-\frac{1}{2} q+i \sqrt[2]{D}}, \quad z_{2}=\sqrt[3]{-\frac{1}{2} q-i \sqrt[2]{D}} .
$$

Therefore,

$$
\begin{aligned}
\left|z_{i}\right|^{2}=\sqrt[3]{\frac{q^{2}}{4}+|D|} & <1+\frac{1}{12} q^{2}+\frac{1}{3}|D| \leq 1+\frac{1}{6} q^{2}+\frac{1}{81}|p|^{3}, \\
\left|z_{i}\right| & <\frac{3}{2}+\frac{1}{12} q^{2}+\frac{1}{162}|p|^{3} .
\end{aligned}
$$

Hence,

$$
\left|x_{i}\right|<3+\frac{1}{6} q^{2}+\frac{1}{81}|p|^{3}
$$

\section{References}

[1] C. M. Doria, The homotopy type of Seiberg-Witten configuration space, to appear in Bol. Soc. Parana. Mat. (2).

[2] A. Gonçalves, Introdução à Álgebra [Introduction to Algebra], Projeto Euclides, vol. 7, Instituto de Matemática Pura e Aplicada, Rio de Janeiro, 1979.

[3] J. Jost, X. Peng, and G. Wang, Variational aspects of the Seiberg-Witten functional, Calc. Var. Partial Differential Equations 4 (1996), no. 3, 205-218.

[4] H. B. Lawson Jr. and M.-L. Michelsohn, Spin Geometry, Princeton Mathematical Series, vol. 38, Princeton University Press, New Jersey, 1989.

[5] A. Marini, Dirichlet and Neumann boundary value problems for Yang-Mills connections, Comm. Pure Appl. Math. 45 (1992), no. 8, 1015-1050.

[6] D. Salamon, Morse theory, the Conley index and Floer homology, Bull. London Math. Soc. 22 (1990), no. 2, 113-140.

[7] K. K. Uhlenbeck, Connections with L $L^{p}$ bounds on curvature, Comm. Math. Phys. 83 (1982), no. 1, 31-42.

[8] E. Witten, Monopoles and four-manifolds, Math. Res. Lett. 1 (1994), no. 6, 769-796.

Celso Melchiades Doria: Departamento de Matemática, Universidade Federal de Santa Catarina, Campus Universitario, Trindade, 88040900 Florianópolis - SC, Brazil

E-mail address: cmdoria@mtm.ufsc.br 